\title{
Q'Hand: A fully automated apparatus for studying haptic processing of spatially distributed inputs
}

\author{
TOM MOORE, MICHAEL BROEKHOVEN, SUSAN LEDERMAN, and SELIM ULUG \\ Queen's University, Kingston, Ontario, Canada
}

\begin{abstract}
Q'Hand is an electromechanical system that permits fully automated preparation and presentation of spatially distributed stimulus displays, as well as on-line data acquisition, all under software control. Selected inputs may be presented to any one or combination of the middle three fingertips of both hands. Both accuracy and reaction times are collected on line. Q'Hand was specifically designed to investigate tactile/haptic preattentive and attentive processing of single and multidimensional stimuli. However, it may also be used to study a variety of other research topics, such as pattern recognition, psychophysical issues, and lateralization of somatosensory function. Further, it may be adapted relatively easily for use in clinical settings. For example, with this apparatus, automated psychophysical tests may be designed for the evaluation of the extent of sensory impairment due to peripheral nerve damage to the hand or to cortical dysfunction.
\end{abstract}

To date, the experimental study of human tactile (cutaneous) and haptic (cutaneous and kinesthetic) perception has been seriously hampered by the dearth of appropriate off-the-shelf technologies. Standard automated equipment is often used in the visual and auditory sciences to prepare and present complex multidimensional displays, as well as to collect data on line. The situation for the tactual sciences is considerably more problematic, however.

We begin by describing the few areas of human tactual perception in which on-line stimulus preparation and/or presentation have proved feasible. First, a number of investigators (e.g., Verrillo, 1968) have used small shaker systems to vibrate different sites on the hand in order to study human temporal sensitivity. With this technology, precise and systematic control of the temporal parameters is possible.

Second, a variety of dynamic vibratory displays have been created, primarily as research tools for studying tactile pattern recognition. For example, the TVSS, or Tactile Vision Substitution System (see, e.g., White, Saunders, Scadden, Bach-y-Rita, \& Collins, 1970) presented

\footnotetext{
The authors wish to express their appreciation for considerable assistance from Cheryl Wilson. They would also like to thank a number of other students and staff for their additional contributions: Sanjay Agrawal, John Glew, Bill Hilthorst, Monica Hurt, Bob Johnston, Allan McPhail, and Scott Young. The project was supported by NSERC Equipment and Operating Grants E0266 and A9854, respectively, and a contract from the Information Technology Research Centre of Excellence (Ontario) to S. J. Lederman. The paper was written with the additional assistance of a grant from the Institute for Robotics and Intelligent Systems Centre of Excellence (Federal). Reprints are available from S. J. Lederman, Queen's University, Department of Psychology, Kingston, Ontario K7L 3N6, Canada. Copies of the copyrighted software and hardware specifications may also be obtained at cost on request.
}

a vibrotactile display to the subject's back. Four hundred solenoid-driven $(400-\mathrm{Hz})$ stimulators were arranged in a $20 \times 20$ matrix, with $12 \mathrm{~mm}$ of separation in both vertical and horizontal directions. Versions of the TVSS have also been developed for presentation to other large skin areas such as the abdomen and forehead, usually under computer control (see, e.g., Cholewiak \& Sherrick, 1981; Janssen, 1983; Shimuzu, Saida, Wake, Nakamura, \& Ohzu, 1982).

Third, dynamic vibrotactile displays have been designed as tactile communication aids for the deaf and blind. For example, a number of vocoders have transformed spoken speech into corresponding spatiotemporal patterns on the skin in real time (see, e.g., Brooks \& Frost, 1983). An artificial electromechanical device has been developed (Reed et al., 1985) for creating multidimensional displays, in an attempt to experimentally evaluate the relative contribution to the deaf-blind Tadoma user of such cues as lip and jaw movements, laryngeal vibrations, and oral airflow. When using the Tadoma method, the listener places his or her hand on the speaker's lower face, using associated articulatory information to track speech. The Optacon, or optical-to-tactile converter (see, e.g., Bliss, 1969) has been developed as a tactile reading device for the blind. A hand-held camera is used to convert print patterns (that differentially activate a $6 \times 24$ matrix of photosensors) into an equivalent vibrotactile pattern on the fingertip, using a $6 \times 24$ matrix of solenoids, driven at $230 \mathrm{~Hz}$. The Optacon has also been successfully adapted for use in basic behavioral and neurophysiological research on the processing of patterns presented to one finger (see, e.g., Craig, 1981; Gardner, 1988; Loomis, 1981) or two fingers (see, e.g., Craig, 1985). It should be noted, however, that with the exception of the specialpurpose Tadoma device, all of the devices above produce 
only one form of stimulus display-that is, spatially and/or temporally distributed vibrotactile patterns.

Next, we consider examples of automated devices that produce or present static stimulus patterns (in contrast to the dynamic displays above). The two discussed here stimulated only a single fingertip. An electromechanical device known as the "tachistotactometer" (Nolan \& Kederis, 1969) was modified by Challman (1978) specifically to study the legibility of braille characters. Any single braille character was produced by raising selected (solenoid-operated) pins from a $3 \times 2$ matrix. Stimulus selection by trial was manually controlled by the experimenter.

A device designed more recently for human psychophysical research by Johnson and Phillips (1981) is arguably the most relevant to our apparatus, although there are also substantial differences, as will be seen. It permitted the application of various micropatterns to a single, stationary finger. A stimulus disk was rotated manually by the experimenter to the desired stimulus on each trial. A linear motor was used to apply that stimulus to the skin, with indentation, force, and duration precisely controlled.

Unfortunately, none of the devices described above is sufficiently appropriate for our specific concerns. ${ }^{1}$ None of them allows us to broadly address issues pertaining to how the human haptic system processes the various object properties to which it is sensitive during purposive manual exploration. All of these properties (relating to surface, material, and structure) are simultaneously available and potentially important to haptic perception. Elsewhere, we have argued that access to multiple sources of information contribute, in large part, to the considerable success with which people can haptically recognize and identify common objects (Klatzky, Lederman, \& Metzger, 1985; Lederman \& Klatzky, 1990).

Our particular goal was to design an apparatus that could be used to address a variety of issues in tactile/haptic preattentive and attentive processing. In our earlier work, we used manual techniques to carry out a haptic variant of the Treisman visual search tasks (see, e.g., Treisman $\&$ Gelade, 1980). For example, manually produced displays consisting of one to six items were presented, also manually, to various finger combinations across one or both hands. (Note that it was necessary for the local inputs to be spatially distributed.) Tasks involved haptic search for single-feature (e.g., "rough"; "vertical"), disjunction (e.g., "rough or vertical"), and conjunction (e.g., "rough and vertical") targets, presented within a background of distractor items (different texture and edgeorientation values). Processing differences were used as a means of selecting a feature set for computationally modelling tactile object recognition (Browse, 1987).

Unfortunately, the manual methods used to prepare and deliver the displays, as well as to collect data, limited the total number of trials in a single session to only about 70 , and the number of replications per stimulus condition to as few as three. Despite the use of within-subject designs, variability was very high (see the System Operating Characteristics section). In comparable visual tasks, at least four times the amount of data has typically been collected. These limitations unfortunately make the study of preattentive and attentive haptic processing with such methods somewhat questionable. Clearly an automated methodology is required.

Q'Hand (Queen's University Apparatus for Hand Research), the apparatus described in this paper, permits fully automated preparation and presentation of spatially distributed stimulus displays, together with on-line data collection, all under software control. Selected inputs (single, multiple dimensions) may be presented simultaneously to any one or combination of the middle three fingers of the left and right hands, thus permitting evaluation of the effects of spatially distributed local inputs. The distance between the two hands is also controllable. The observer may freely explore the display, following contact. Both accuracy and reaction times are collected on line through custom software that reads time to close one of two thumb switches, or through voice input. With this apparatus, as many as $\mathbf{4 0 0}$ trials may be collected within a single session, constituting a 4- to 14-fold increase in a subject's database, depending on the experiment (cf. Lederman, Browse, \& Klatzky, 1988). With substantial reduction in reaction time variability, it is possible to systematically address a number of issues that pertain to haptic preattentive and attentive processing of single and multidimensional stimuli.

The current apparatus may also serve a number of additional functions, provided that control is not required. For example, standard psychophysical experiments that address single-finger perception or recognition of surface textures, compliances, thermal properties, and micropatterns may all be performed with Q'Hand by mounting the relevant set of stimuli on a single stimulus drum. Q'Hand may be used to examine a variety of issues regarding the lateralization of somatosensory function, by presenting any input unimanually or dichhaptically (i.e., simultaneous inputs to both hands: Witelson, 1974). Finally, Q'Hand may be adapted relatively easily for the systematic assessment of patients who have sustained neurological damage (central or peripheral nerve impairment to the hand). Quick and systematic psychophysical tests may be developed to assess the extent to which a patient can perceive object properties such as those mentioned above with any targeted finger (for further discussion, see Lederman \& Klatzky, 1987).

The apparatus was designed with the following goals in mind:

1. We wanted to be able to prepare stimulus displays rapidly on line by means of software control. The display could consist of one to six items, presented simultaneously to the middle three fingertips of one or both hands.

2. Presentation of the stimulus display to the fingertips had to be automated and fast.

3. The subject's fingertips should be positioned at the start of each trial so that the stimulus items in the display 
would contact predesignated fingers at approximately the same time.

4. The spatial separation between the two hands could be manipulated.

5. We desired a maximum thickness of $3 \mathrm{~cm}$ for stimulus items, to permit production of stimuli with substantial variation in the third dimension.

6. The cost of the device must be kept to a minimum, through the utilization of readily available standard components, materials, and machine shop tools and techniques.

The Queen's University Apparatus for Hand Research was designed and built to meet these requirements. In the remainder of this paper, we will describe the basic design and operating principle of Q'Hand. Because those interested in constructing such a system might wish to impose certain design/software modifications of their own, we make no attempt to provide explicit hardware specifications/software here. The interested reader should contact S. J. Lederman for design drawings and copyrighted software, which may be obtained at cost on request.

\section{HARDWARE}

Q'Hand consists of three major components, as shown in the schematic presented in Figure 1.

The presentation platform is the mechanical device that presents the stimulus display to the subject's fingertips. The motor control cabinet contains the electronics and associated power supply necessary to operate the stepper motors employed on the presentation platform. The microcomputer provides both the low-level routines required to control the stepper motors and the high-level routines

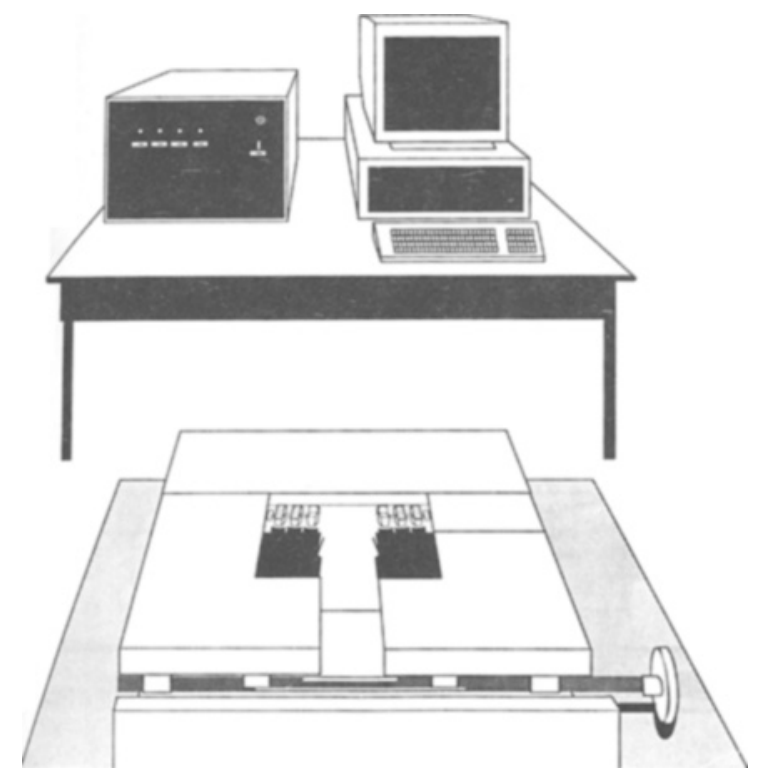

Figure 1. Q'Hand: Schematic of the overall system consisting of the presentation platform, the motor control cabinet, and the microcomputer. that permit the rapid development of an ordered set of stimulus displays through experiment-specific software. The microcomputer also provides necessary timing and data archiving functions.

\section{Presentation Platform}

The presentation platform (Figure 2) consists of a simple wooden cabinet that incorporates opaque Plexiglas palm and adjustable finger supports. The cabinet has an open slot in the top, through which the stimulus displays are presented to the fingertips. The actual mechanical details of the presentation device have been deliberately hidden from view to minimize the subject's possible apprehension.

Figure 3 shows a simplified drawing of the mechanical components contained within the cabinet. A coordinate system is shown for convenience in the description. The $x y$ plane is horizontal and contains the base plate.

The aluminum base plate acts as a support for all other details. The plate is $60 \times 55 \mathrm{~cm}$ and $.6 \mathrm{~cm}$ thick.

The lift motor (SloSyn M111-FD-302 stepper motor) acts through a gear reduction and chain mechanism to simultaneously drive two parallel shafts. On each shaft, there are two identical cams, each of which supports one side of the Plexiglas lift platform. Thus, as the stepper motor operates, the cams are turned synchronously and the lift platform is raised, while remaining horizontal, through a distance equal to the cam throw.

The full "up" position is used to present the stimulus display to the subject's fingertips, while the full "down" position is used to reorder the individual stimulus items in the display prior to subsequent presentations. The main platform may be positioned anywhere between these extremes, as required, through software control. This permits some flexibility regarding stimulus height, without one's having to adjust the dimensions of the stimuli themselves.

On the main platform, there are two Plexiglas auxiliary platforms mounted in a manner that allows independent positioning in the $y$ direction. On each auxiliary platform, there is a group of three stimulus drums and their respective indexing motors (Airpax 4SH-12A56S stepper motors). Each stimulus drum and its indexing motor are mounted on a separate aluminum slide plate, which permits independent positioning in the $x$ direction.

Thus, the auxiliary platforms may be adjusted to permit variation of the spatial separation between the hands. The position in the $x$ direction for each stimulus drum may be adjusted as well, to accommodate subject variations in fingertip position.

The stimulus drums are mounted on spindles with ball bearings, so they turn easily. Each drum has been machined to provide eight facets with equal $3.2 \times 2.2 \mathrm{~cm}$ dimensions. On each of these facets, a stimulus may be mounted. At present, each of the six stimulus drums has had two adjoining facets machined away, as shown in Figure 3 . This was done to provide a no-stimulus condition, to indicate when there is nothing contacting a fingertip 


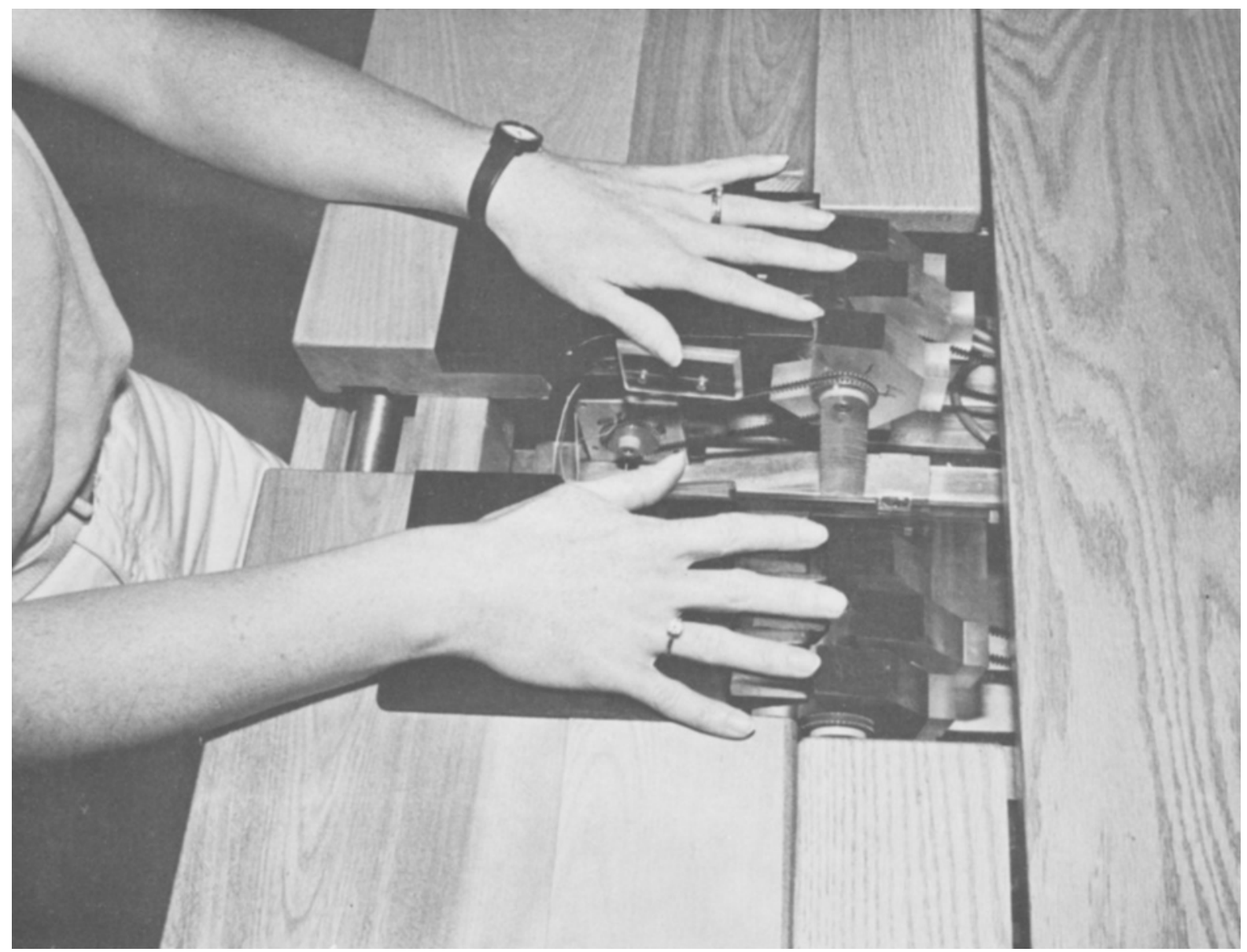

Figure 2. Photograph of presentation platform, showing subject's hands resting on hand/finger rests. The hands are pulled back to allow a view of the drum facets, to which stimuli (not shown) are attached.

for a stimulus display. The drums can easily be replaced with drums containing the full eight facets, or more if desired (with some simple modifications).

Each stimulus drum can be turned by its indexing motor with a toothed, reinforced rubber drive belt and sprockets. Due to the relatively high available starting torque of the motor and the low drum inertia and bearing friction, a drive ratio of $1: 1$ was used. The holding torque for each indexing motor is $80 \mathrm{oz}-\mathrm{in}$. This is sufficient to prevent any movement of the drum under the influence of the fingertip/stimulus interface forces generated during a trial.

The two Plexiglas hand-rest assemblies support the subject's hands and also position the fingertips. The separation between the two hands can be adjusted by turning the hand-rest adjustment screw.

The fingers are supported by finger rests that are individually adjustable, so that raised markers (inserted in the distal tip of each finger rest) may be positioned beneath the subject's distal joints. This provides an optional method of positioning the hands with respect to the stimu- lus display. Once the positions of the finger rests have been calibrated for a given subject, the raised markers permit only the distal segment of each finger to project beyond the finger rest.

Each hand rest incorporates a thumb-operated paddle switch, which can be used to signal the completion of a given task. Such information may be processed in various ways, depending on the particular experimental design (e.g., the two thumb switches may be used to designate "yes" and "no" responses).

As an alternative response, a voice-activated switch can be closed to record verbal reaction times.

\section{Motor Control Cabinet}

The motor control cabinet contains all the electronic controls for the stepper motors and a mains-operated switching power supply (Figure 4).

Each of the six indexing motors is controlled by its own commercially available driver board (AMSI Corp., 5005DB). The inputs to the driver board are both TTL- and CMOS-compatible, which assures direct compatibility 


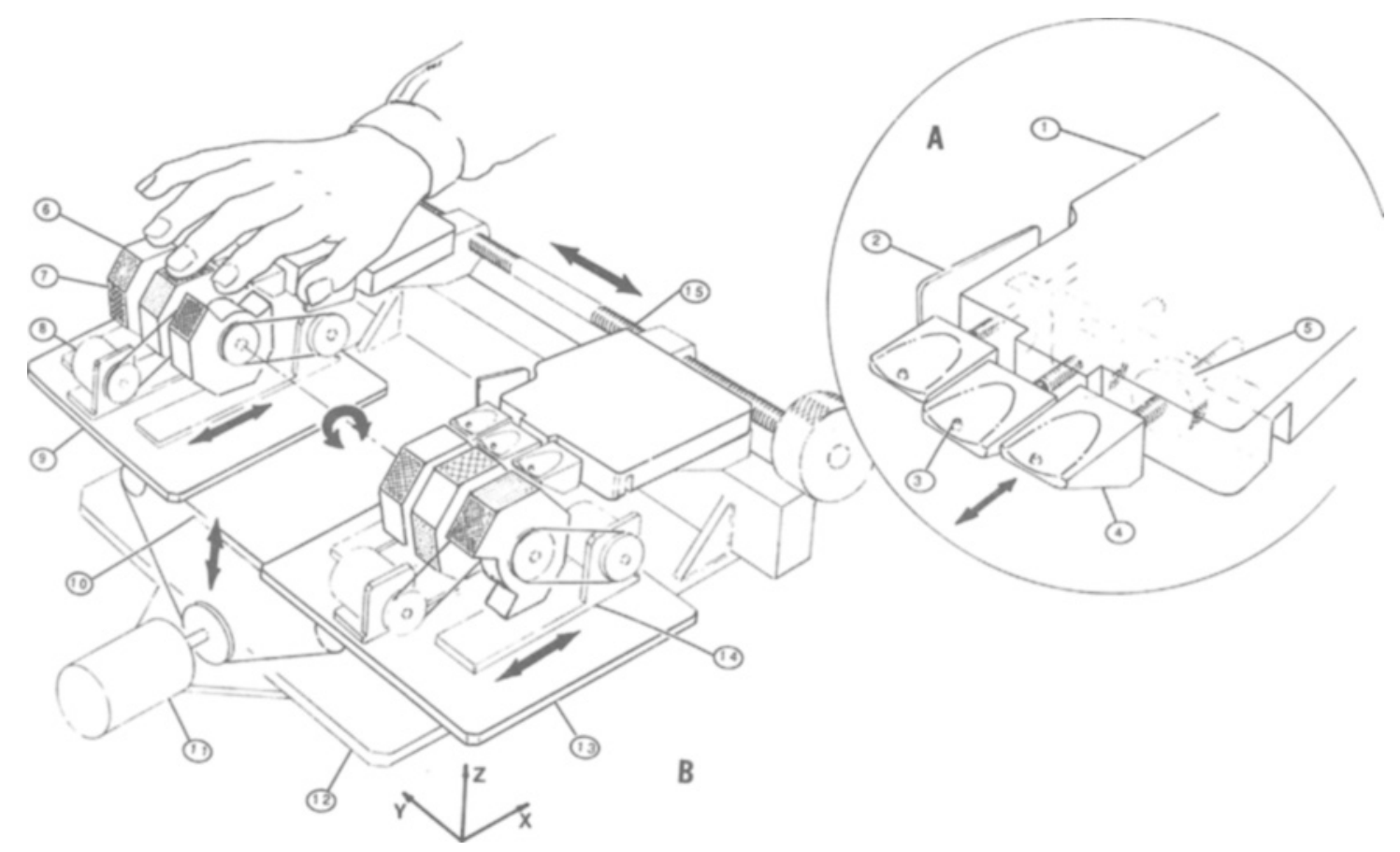

Figure 3. Schematic of the mechanical components contained within the wooden cabinet. (A) Enlarged view of hand/ninger rest: (1) hand rest, (2) thumb switch, (3) finger-position marker, (4) finger rest, (5) finger-rest adjustor. (B) View of entire assembly: (6) stimulus drum, (7) facet (with stimulus), (8) indexing motor, (9) right auxiliary platform, (10) lift platform, (11) lift motor, (12) base plate, (13) lef audiliary platform, (14) slide plate, (15) hand-rest adjustment screw.

with virtually any microcomputer-based digital input/output (I/O) board. In addition, the inputs are optically isolated, thus affording maximum protection from stepper motor-generated electrical noise. It should be noted that the stepper motors are used in an open-loop control configuration. The position of the driven devices is simply inferred from the number of step commands sent to the motors. This lack of position feedback information was considered acceptable in view of the relatively low motor stepping rates and torque requirements. The likelihood of a "loss of steps" was very remote and did not justify the added complexity and expense of closed loop control. Experience has confirmed the validity of this approach.

The motion of each motor is controlled by two bits of the parallel digital I/O port. One bit is connected to the "trigger" input of the driver board. When this bit is toggled between logical " 1 " and logical " 0 ," the stepper motor is rotated one step (i.e., $1.8^{\circ}$ ). The stepping rate is thus controlled by the pulse rate applied to the input trigger, and the number of steps taken is determined by the total number of pulses. The other bit is connected to the "rotation" input of the driver board. If this bit is set to logical " 1 ," ' rotation is clockwise; if it is set to logical " 0 ," rotation is counterclockwise. Because 2 bits are required to control each indexing motor, 12 bits of the digital I/O port are dedicated to this task.

The lift motor is also controlled by a commercially available driver board (SloSyn STM 101). In this case, however, there are two separate trigger inputs. Toggling from logical " 1 " to logical " 0 " produces one step of the motor $\left(1.8^{\circ}\right)$. The direction of rotation is then determined by whichever of the two trigger inputs one uses.

Thus the total number of bits of the digital $1 / 0$ port dedicated for motor control is 14 . Note that this number could have been reduced to $8 \mathrm{had}$ all the motors been restricted to unidirectional rotation. However, it was decided to retain the option of employing bidirectional motion for each motor, since it would provide greater flexibility, and I/O bits were available.

\section{SOFTWARE}

\section{Machine Dependencies}

The digital $\mathrm{I} / \mathrm{O}$ for the electronic interface is done via a Data Translation DT2817 board. This is a 4-byte interface, driven at $5 \mathrm{~V}$, requiring an 8-bit, standard PC bus slot. Any machine that is to run this software directly requires this board. Should another board be used, some software modification might be required.

The timing is completed via DOS interrupt 8, modified to produce an interrupt more than 1,000 times per second. This will run on any IBM-compatible machine running MS-DOS Versions 2.1-3.3. If translation to another machine is required, a similar routine must be written that can time events to an accuracy of $1 \mathrm{msec}$.

The software is written in Turbo C, Version 2.0 (for further details, see the Implementation section). No libraries unique to this compiler were used. The code can 


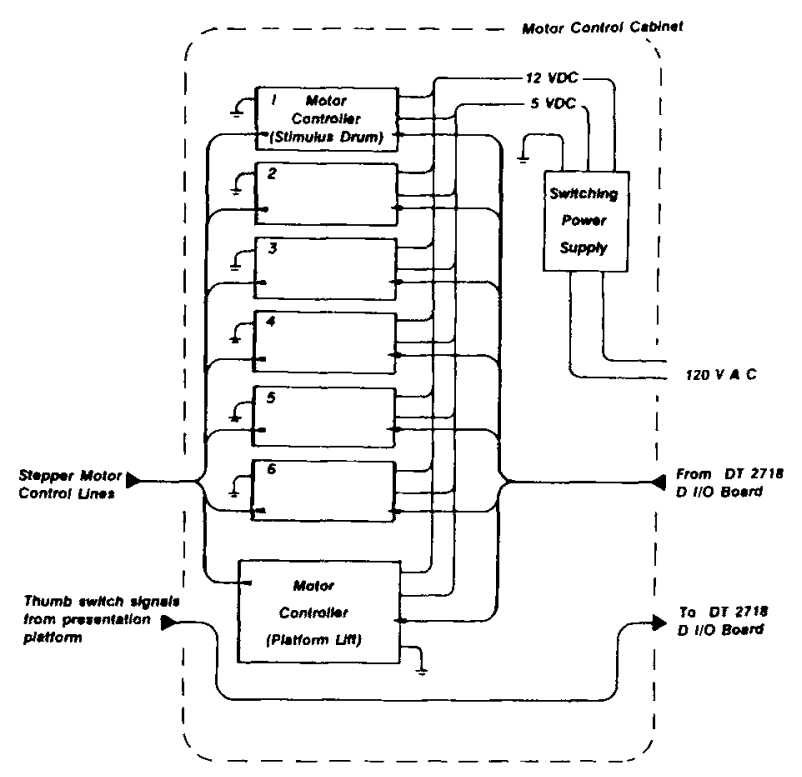

Figure 4. Schematic showing electronic control components.

easily be moved to another $C$ compiler supporting the ANSI standard.

\section{Overview}

The software component of the Q'Hand system is needed to control specific functions of an experiment. It is designed as a modular subsystem that can be adapted easily to many tasks. The software divides into three distinct sections.

The lowest level consists of the routines that physically drive the apparatus. These routines access the ports of the digital I/O board in the computer and perform the lowlevel operations for sending the appropriate pulses to the Q'Hand motor control cabinet.

The next highest level, which surrounds the low-level routines, presents specific trials to the subject and gathers certain information (e.g., reaction time). Routines that provide menus for modification of certain Q'Hand operating parameters are also enclosed in this level (see section below on Q'Hand control functions), but they will not be discussed here. The low and middle level of software are collectively known as the "utility" routines.

The highest software level is used to prepare a sequence of trials (stimulus displays) for a given experiment. It also records this information on disk, along with the subject's responses, for subsequent data analysis. This code is specific to the experiment, and it will likely have to be modified for different types of experiments. In contrast, the middle level must change only as the desired information changes (e.g., the dependent variable). The lowlevel utility routines will not change with experiments.

Implementation. All the software is currently implemented in the $C$ language (Turbo $C$, Version 2.0).
Parts were originally written in Pascal (Turbo Pascal, Version 3.0) and then ported to $C$. The philosophy behind the utilities is to provide a set of modular routines that are callable from a $C$ program. To complete a new experiment, a software program would need to be written that would implement these utilities appropriately. Thus, the operation of Q'Hand can be reliably controlled without the programmer's knowing the details of its operation. By writing code that sets up trials according to the protocol, one can use Q'Hand as an effective experimental tool with little difficulty.

High-level software functions. This paper covers in detail only the utility routines for Q'Hand, which are intimately tied to the operation of the device. High-level software has been implemented, but it will not be discussed extensively here. The operation of the high-level code is briefly outlined in Table 1 .

QHand control functions. A global structure variable, called "status," contains flags and variables that control certain aspects of Q'Hand's operation. Referred to as the "status parameters," these flags can be modified from menus during the course of an experiment and can retain their values permanently on disk. Table 2 lists the Q'Hand operations controlled by the status parameters.

Utility software. The utility software consists of three major sections. Each section, which contains drivers for a specific part of Q'Hand, will be discussed in turn.

Drum rotation. Routines to drive the six stimulus drums of Q'Hand compose the first section of the utilities. A status parameter controls whether the six drums rotate at a single or variable speed. A single speed may be chosen when it is desirable to rotate all drums at the same rate; in this case, the drums will end rotation at different times, depending on the amount of rotation required to rotate the drum to the designated facet. A variable speed rotation may be chosen to start and stop all drums at the same time. In the second case, the six drums all turn for the same length of time, with each drum necessarily rotating at a different velocity. We currently prefer the latter, because it lowers the noise level of Q'Hand's operation-selecting an empirically determined optimum speed reduces resonance in the stepper motors. We have also confirmed that auditory cues associated with varying drum movement could not be used to predict the nature of the next stimulus display.

Table 1

Operation of the High-Level Software Code

Permits experimenter to test the proper operation of thumb switches and voice sensor for each subject before the experiment commences

On-line control of individual drum and platform

Builds and randomizes a sequence of trials in the order determined by the experiment protocol

Calls the utility routines to present the trials to the subject

Measures and records in a datafile on disk all relevant information about a trial, including stimulus pattern, reaction time, and so forth Allows interface to all above functions through convenient menus 
Table 2

Q'Hand Operations Controlled by Status Parameters

Stimulus drum speed

Platform speed

Distance platform moves

Single or variable speed drum rotation

Voice or thumb-switch triggering

Window of time in which both thumb switches cannot be depressed (to discount trials in which the subject depressed both paddles accidentally)

Time delay between individual trials to allow the subject to reorient hands

Acceptable minimum and maximum reaction times (If a subject reacts outside of this window, the trial is considered invalid. The values of these times are set by experiment-specific software.)

Function of thumb switches (The orientation of this flag determines which thumb switch indicates "yes" and which one indicates "no.")

Two routines access the stimulus drums. The first has the $\mathrm{C}$ function prototype below:

stimuli(int steps[6], int delays[6], int directions[6])

This routine accepts the number of steps for each drum, the delay between steps for each drum, and the direction in which each drum will rotate (clockwise or counterclockwise). It generates a sequence of output steps for the stepper motors, which ensures that all drums move their respective steps with their respective delays between steps. It then feeds the sequence out through the computer ports to the motor control cabinet. The delays allow the calling routine to control the speed of the individual drums.

The second routine that controls the drums is slightly higher in level; this is the one typically called from an experiment program. It has the $\mathrm{C}$ function prototype below:

$$
\text { Position_stimuli (int position[6]) }
$$

This routine takes an array of six integer arguments, one for each drum. The values for each may take any number between 0 and 7 , representing the eight facets of the drum. The routine assumes that at the start of the experiment, the drums are positioned on Facet 0 (this may be any facet; it is used as a frame of reference for the software and the experimenter). Using static variables, the routine remembers which facet each drum is positioned on at any given time, and it uses this information to position the drums on any new facet. When a high-level piece of code for an experiment determines which facets compose a stimulus display, it can reference each facet by its number. It then passes these numbers to Position —stimuli( ), which determines each drum rotation. Position_stimuli( ) calls stimuli( ). If the status parameters indicate that variable speed rotation is to be used for the drums, Positionstimuli( ) calculates the necessary delays to ensure that the six drums start and stop rotating simultaneously. Position _stimuli( ) ensures that all drums rotate in the same direction, away from the subject's fingers.

Platform motion. Two routines effect platform motion. The first routine moves the platform with a delay between each step. The direction parameter controls the orienta- tion (clockwise or counterclockwise) of motion of the platform cams:

Move_platform (int steps, int delay, int direction)

The second routine is called by the middle-level experimental software. Using static variables, it remembers the position of the platform from the last move. The parameter indicates whether the desired position for the platform is up or down. If the platform is already in that position, no motion occurs. The speed and the number of steps through which the platform moves are determined by the status parameters, allowing the experimenter to fine tune how the platform rises up to contact the subject's fingers. The number of steps the platform moves must be changed as the height of the stimulus mounted on the drums changes:

\section{Platform (int direction)}

Trigger detection. One of two methods of triggering can be selected. If voice triggering is used, the experimenter must be present at the computer keyboard to indicate the correctness of the subject's response. If thumb-switch triggering is used, the subject can directly indicate a binary response by activating the appropriate thumb switch. Sensor triggering therefore eliminates the need for experimenter input.

Voice triggering. The routine to read the voice port has the following prototype:

$$
\text { void get_response_voice(int *response) }
$$

This routine checks the value of the port for the voice sensor line and returns a value representing "on" or "off" via the parameter response. Attached to the mo-

Table 3

\begin{tabular}{|c|c|}
\hline Feature & Performance \\
\hline On-line display preparation time & $1.32 \mathrm{sec}$ \\
\hline Duration of platform lift/drop cycle & $1.31 \mathrm{sec}$ \\
\hline Minimum contact time (mean and SD) & $117 \pm 48 \mathrm{msec}$ \\
\hline $\begin{array}{l}\text { Temporal asymmetry in contacting all } \\
\text { pairs of fingers }(M \text { and } S D)\end{array}$ & $10 \pm 20 \mathrm{msec}$ \\
\hline $\begin{array}{l}\text { Mean response time to close } \\
\text { thumb switch }\end{array}$ & $100 \mathrm{msec}$ \\
\hline $\begin{array}{l}\text { Time to read input from } \\
\text { thumb switch }\end{array}$ & $\begin{array}{l}\text { order of } 10 \text { s of } \\
\text { microseconds }\end{array}$ \\
\hline $\begin{array}{l}\text { Range of motion ( } x \text {-axis) of adjustable } \\
\text { finger rests }\end{array}$ & $14 \mathrm{~mm}$ \\
\hline $\begin{array}{l}\text { Adjustable range of separation } \\
\text { between homologous fingers of two } \\
\text { hands (range: thumb switches }\end{array}$ & \\
\hline $\begin{array}{l}\text { touching-maximum separation) } \\
\text { Maximum stimulus dimensions }\end{array}$ & $11-30 \mathrm{~cm}$ \\
\hline $\begin{array}{l}\quad(x-, y-, z \text {-axes }) \\
\text { Estimated number of trials/hour }\end{array}$ & $3.2 \times 2.2 \times 3.0 \mathrm{~cm}$ \\
\hline (includes 70 practice trials) & approximately 400 \\
\hline $\begin{array}{l}\text { Clock accuracy } \\
\text { Standard error re: co }\end{array}$ & $1 \mathrm{msec}$ \\
\hline experiment with manual apparatus & $>5$-fold decrease \\
\hline
\end{tabular}

Operating Performance of Q'Hand 
tor control cabinet, the voice port is connected to a microphone that the subject uses to indicate a response. This routine is called from Reaction time( ) (see below).

Thumb-switch triggering. The routine to read the sensor port has the prototype below:

$$
\text { void get__response_sensor(int * response) }
$$

This routine reads the status of the paddle switches on the Q'Hand hand rests. The parameter response can return the values open, left closed, right closed, or both closed. The routine reads the values of the two sensor lines. If one goes low (they are 5-V active low signals), the circuit is closed. This indicates that the subject pressed one of the switches. The routine then waits for a period of time to see if the other line also closes. If so, the routine returns the value corresponding to both closed. If the situation is normal (i.e., only one line is closed), the appropriate return value (left or right) is given. If neither line is closed, the routine returns, indicating that no trigger was given. A status parameter determines the period of time that the software waits to detect a second closing circuit (typically $50 \mathrm{msec}$ ).

This routine is called from Reaction _time( ). It is also called from the experiment software to determine the status of the sensor lines at the beginning of a trial. If a subject closes a circuit prematurely (i.e., before processing could possibly begin), the stimulus condition is repeated according to rules set in the experiment software. The selection of a minimum reaction time is experimenterdetermined.

The routine Reaction _time( ) has the following prototype:

unsigned long Reaction_time (int $*_{r t}$ _code)

It is called to measure reaction time, after the stimulus display has been presented. It returns the amount of time (in milliseconds) that the subject takes to trigger a response. The parameter $\mathrm{rt} \_$code is a return value indicating the nature of the triggering. In the case of voice-key triggering, $\mathrm{rt}$ code is meaningless. With sensor triggering, it returns a value representing "yes," "no," or invalid. A status parameter determines which of the binary responses (e.g., "yes" or "no") a closed switch represents. An invalid condition indicates that both switches were closed (the interpretation of this condition is specific to the experiment).

\section{SYSTEM OPERATING CHARACTERISTICS}

Selected operating features of the system are presented in Table 3. Further details concerning the methodologies used to determine performance are presented in this section.

To measure the time to prepare a single display on line, we first calculated the mean time to prepare a single display, deriving the latter from the time it took to prepare a set of $\mathbf{3 6}$ different stimulus displays with no interven- ing delays. The mean of 10 such readings was used as the estimate of on-line stimulus preparation time.

A similar procedure was used to determine the average duration of a single platform lift-drop cycle, with 36 continuous cycles replacing stimulus displays in the description above.

Time to contact was simultaneously measured for each finger by covering both surfaces of each of the six finger/ drum facet combinations with very thin aluminum shims. The timer was activated when the platform began its lift motion, and it was stopped by the contact, which closed an electrical circuit. The average time to contact was based on 150 trials for each of three subjects. The latter were instructed to reposition their fingers following each trial. The minimum contact time was measured as the mean difference between times at which contact was made and subsequently broken.

The assessment of temporal contact asynchrony was calculated as the average temporal difference between all finger pairs. This constitutes a change of $1 \%-4 \%$ in the baseline reaction time for processing a single item (given that baseline reaction times have ranged from approximately 250 to $950 \mathrm{msec}$ in unpublished data involving different texture-search conditions collected with Q'Hand). We recognize that when active exploration is permitted, and when stimuli vary in thickness or compliance, for example, the level of precision in timing will likely decrease. Unfortunately, this tradeoff between control and ecological validity is an inevitable problem in the experimental study of behavior.

The time to close the thumb switch was based on data obtained from 6 subjects. Each subject was presented with 12 displays, consisting of from one to six Plexiglas blocks randomly assigned to fingers. The subjects were instructed to press the designated thumb switch (left or right) as soon as the display contacted their fingers. Response times for six left- and six right-handed responses were averaged to provide an estimate of each subject's motor response time.

The average variability (standard error) of data collected with Q'Hand was compared with that obtained in a comparable single feature search experiment, using the manual apparatus described in Lederman et al. (1988).

\section{SUMMARY}

Q'Hand was custom-designed to permit fully automated preparation and presentation of stimulus displays to designated fingertips of both hands. Inasmuch as the apparatus further allows on-line data acquisition (accuracy and reaction times), a considerable amount of data may be collected in a single session, thereby reducing within-subject data variability. Q'Hand may be used to study a variety of topics that pertain to tactile and haptic processing, such as preattentive and attentive processing, pattern recognition, tactual psychophysics, and lateralization of somatosensory function. It may also be adapted with relative ease for use in clinical settings. 


\section{REFERENCES}

Buss, J. C. (1969). A relatively high-resolution reading aid for the blind. IEEE Transaction on Man-Machine Systems, 10, 1-9.

Brooks, P. L., \& Frost, B. J. (1983). Evaluation of a tactile vocoder for word recognition. Journal of the Acoustical Society of America, 74, 34-39.

Browse, R. A. (1987). Feature-based tactile object recognition. IEEE Transactions on Pattern Analysis \& Machine Intelligence, 9, 779-786.

ChallmaN, B. E. (1978). Variables influencing the identification of single braille characters. Unpublished master's thesis, University of Louisville.

Cholewiak, R. W., \& Sherrick, C. E. (1981). A computer-controlled matrix system for presentation to the skin of complex spatiotemporal patterns. Behavior Research Methods \& Instrumentation, 13, 667-673.

CraIG, J. C. (1981). Tactile letter recognition: Pattern duration and modes of pattern generation. Perception \& Psychophysics, 30, $540-546$

CralG, J. C. (1985). Attending to two fingers: Two hands are better than one. Perception \& Psychophysics, 38, 496-511.

GARDNER, E. P. (1988). What the robot's hand should tell the robot's brain: Feature detection in a biological neural network. Proceedings of the IEEE Intemational Conference on Neural Networks, 2, 557-565.

JANSSON, G. (1983). Tactile guidance of movement. International Journal of Neuroscience, 19, 37-46.

Johnson, K. O., Phillips, J. R. (1981). Tactile spatial resolution: I. Two-point discrimination, gap detection, grating resolution, and letter recognition. Joumal of Neurophysiology, 46, 1177-1191.

Johnson, K. O., \& Phillips, J. R. (1988). A rotating drum stimulator for scanning embossed patterns and textures across the hand. Joumal of Neuroscience Methods, 22, 221-231.

Klatzky, R. L., Lederman, S. J., Metzger, V. A. (1985). Identifying objects by touch: An "expert system." Perception \& Psychophysics, 37, 299-302.

LAMotte, R. H., Whitehouse, G. M., Robinson, C. J., \& DAvis, F. (1983). A tactile stimulator for controlled movements of textured surfaces across the skin. Joumal of Electrophysiological Technology, 10, 1-17.

Lederman, S. J., Browse, R. A., Klatzky, R. L. (1988). Haptic processing of spatially distributed information. Perception \& Psychophysics, 44, 222-232.
Lederman, S. J., \& KLATzky, R. L. (1987). Hand movements: A window into haptic object recognition. Cognitive Psychology, 19, 342-368.

Lederman, S. J., \& KlatzKY, R. L. (1990). Haptic exploration and object representation. In M. A. Goodale (Ed.), Vision and action: The control of grasping (pp. 98-109). Norwood, NJ: Ablex.

Loomis, J. M. (1981). On the tangibility of letters and braille. Perception \& Psychophysics, 29, 37-46.

Nolan, C. Y., \& Kederus, C. J. (1969). Perceprual factors in braille word recognition. New York: American Foundation for the Blind.

Reed, C. M., Rabinowitz, W. M., Durlach, N. I., Braida, L. D., Conway-Fithian, S., \& Schultz, M. C. (1985). Research on the Tadoma method of speech communication. Joumal of the Acoustical Society of America, 77, 247-257.

Shimizu, Y., Saida, S., Wake, T., Nakamura, A., * OhzU, H. (1982). Optimum design of tactile display for a reading aid. In J. Raviv (Ed.), Uses of computers in aiding the disabled. Amsterdam: NorthHolland.

Treisman, A., * Gelade, G. (1980). A feature integration theory of attention. Cognitive Psychology, 12, 97.136.

VERRILLO, R. T. (1968). A duplex mechanism of mechanoreception. In D. Kenshalo (Ed.), The skin senses (pp. 112-156). Springfield, IL: Thomas.

White, B. W., Saunders, F. A., Scadden, L., Bach-y-Rita, P., * Colluns, C. C. (1970). Seeing with the skin. Perception \& Psychophysics, 7, 23-27.

Witelson, S. F. (1974). Hemispheric specialization for linguistic and nonlinguistic tactual perception using a dichotomous stimulation technique. Cortex, 10, 3-17.

\section{NOTE}

1. Here we do not describe equipment used to measure single-unit responses by the somatosensory system to vary fine textures and micropatterns. Typically, a single stimulus is moved through a unit's receptive field (see, e.g., Johnson \& Phillips, 1988; LaMotte, Whitehouse, Robinson, \& Davis, 1983). Such devices are sufficiently dedicated to neurophysiological and corresponding psychophysical functions that they would be difficult to modify for the multifingered spatiotemporal haptic tasks discussed in the current paper.

(Manuscript received July 31, 1990; revision accepted for publication December 4, 1990.) 